\title{
95. NOTAS SOBRE LA VEGETACIÓN DE ANDALUCÍA. V.
}

\author{
Andrés V. PÉREZ LATORRE y Baltasar CABEZUDO
}

Short notes about Andalusian vegetation. $V$.

Palabras clave. Vegetación, sintaxonomía, Pinguicula, Cistus laurifolius, Andalucía, España.

Key words. Vegetation, syntaxonomy, Pinguicula, Cistus laurifolius, Andalusia, Spain.

\section{Pinguiculion longifoliae F. Casas 1970}

El género Pinguicula está representado en el Parque Natural de las Sierras Tejeda, Almijara y Alhama (Málaga-Granada) por dos especies según Blanca et al. (1999). P. dertosensis (Cañig.) Mateo \& M. B. Crespo aparece en dos localidades, una de ellas en Sierra Tejeda (Alhama de Granada) y otra en Sierra Almijara (Arenas del Rey), ambas de la provincia de Granada. P. vallisneriifolia Webb está citada para Jete (también Granada, Sierra de Cázulas).

Nieto Caldera (1987) ya había localizado las dos primeras localidades y las había asignado a $P$. vallisnerifolia, proponiendo el EucladioPinguiculetum vallisneriifoliae (Díaz et al., 1982) en una tabla con inventarios de Sierra Tejeda (Alhama de Granada), de Sierra de Cazorla y de Sierra de Alcaraz de donde es el holotypus. Como en dicha tabla se habían reunido tres especies de Pinguicula distintas (ver Blanca et al., 1999) (invs. 1 a $3=P$. dertosensis, inv. $4=P$. vallisneriifolia $\mathrm{e}$ invs. 5 a $8=P$. mundi) era necesaria una reestructuración sintaxonómica.

El inventario 4 corresponde al Pinguiculetum vallisneriifoliae ya descrito por Heywood (1953) y validado por Varo y Fernández Casas (1970). Por otro lado, en Rivas Martínez et al. (2002:234) aparece una corrección de Asensi \& Díez donde aluden a la tabla de Díaz et al. (op. cit., invs. 5 a 8) que pasan a ser Eucladio-Pinguiculetum mundi, que incluye el tipo de la asociación de Díaz et al. (art. 40b). En dicha obra (2002:208), y al no comprender el tipo de la asociación original EucladioPinguiculetum vallisneriifoliae, también proponen como nueva (art. 15, art. 24) SouthbyoPinguiculetum dertosensis transcribiendo el inventario número 3 de dicha tabla de Díaz et al. (op. cit) aunque sin explicitar la autoría del inventario en el campo (recomendaciones 39A ó 46D).

Por tanto la sintaxonomía de las comunidades del Pinguiculion longifoliae en Tejeda-AlmijaraCázulas quedaría hasta el momento como sigue:

ADIANTETEA CAPILLI-VENERIS Br.-Bl. in Br.-Bl., Roussine \& Nègre 1952

+ Adiantetalia capilli-veneris Br.-Bl. ex Horvatiç 1939

* Pinguiculion longifoliae F. Casas 1970

1. Southbyo tophaceae-Pinguiculetum dertosensis Asensi \& Díez in Rivas Martínez et al. 2002.

[= Eucladio-Pinguiculetum vallisneriifoliae Díaz, Guerra y Nieto 1982 pp., tabla 1 invs. 1 a 3. (arts. 15 y 24)]

2. Pinguiculetum vallisneriifoliae Heywood ex Varo \& F. Casas 1970

[syn. Eucladio-Pinguiculetum vallisneriifoliae Díaz, Guerra y Nieto 1982 pp., tabla 1, inv. 4].

Por último reseñar que la población de $P$. vallisneriifolia que hemos localizado en la zona SE del Parque se encuentra en el municipio de Otívar y no en el de Jete como se indica en Blanca et al. (1999) (pliego GDAC 37737 R. Zamora); desconocemos si se trata de una localidad distinta o de un error geográfico, por la escasez de biotopos potenciales para Pinguicula en Jete.

Cistion laurifolii Rivas Goday in Rivas Goday et al. 1956 em. Rivas Martínez 1979

Los singulares jarales sobre sustratos descarbonatados (calizas y dolomías) del sector Rondeño (Sierra de las Nieves) fueron estudiados 
fitosociológicamente por Pérez Latorre et al. (1998) adscribiéndolos a una comunidad de Cistion laurifolii (Cisto-Lavanduletea). Este jaral mesomediterráneo aparece en zonas con ombroclima húmedo. Es una formación de matorral caracterizada por Cistus laurifolius que aparece aisladamente en el areal de los encinares y pinsapares calcícolas debido a la aparición de calizas y brechas descarbonatadas o pequeños afloramientos silíceos. Especies frecuentes en esta comunidad son Cistus crispus, Cistus ladanifer, Lavandula stoechas, Erica australis, Erica arborea y Ulex baeticus. Se desarrolla sobre suelos degradados y es de distribución rondense. Constituye una de las etapas seriales de los encinares con alcornoques del Paeonio-Querceto rotundifoliae $S$ y, muy rara vez, de los pinsapares de Paeonio-Abieteto pinsapo $S$.

Cano et al. (2003) describen la nueva subasociación (Ulici baetici-Lavanduletum lanatae lavanduletosum stoechadis) que creemos que es la misma comunidad de Cistus laurifolius que propusieron Pérez Latorre et al. (1998), obra a la

\begin{tabular}{|c|c|c|c|}
\hline INVENTARIO N ${ }^{\circ}$ & 1 & 2 & A \\
\hline Orientación & SSE & $\mathrm{S}$ & - \\
\hline Inclinación $\left({ }^{\circ}\right)$ & 25 & 20 & - \\
\hline Altitud $(\mathrm{m})$ & 1200 & 1200 & - \\
\hline Cobertura $(\%)$ & 100 & 100 & - \\
\hline Área (m 2) & 200 & 200 & - \\
\hline Altura vegetación (cm) & 150 & 250 & - \\
\hline \multicolumn{4}{|l|}{ Características de comunidad } \\
\hline Erica australis & 3 & 3 & $x$ \\
\hline \multicolumn{4}{|l|}{ Características de Cisto-Lavanduletea } \\
\hline Cistus crispus & + & . & . \\
\hline Cistus ladanifer & 2 & . & $\mathrm{x}$ \\
\hline \multicolumn{4}{|l|}{ Características de Rosmarinetea } \\
\hline $\begin{array}{l}\text { Dorycnium pentaphyllum } \\
\text { Staehelina dubia }\end{array}$ & 1 & $\cdot$ & $\cdot$ \\
\hline Teucrium similatum & $\begin{array}{l}+ \\
+\end{array}$ & $\dot{r}$ & $\cdot$ \\
\hline Lavandula lanata & . & . & $\dot{x}$ \\
\hline \multicolumn{4}{|l|}{ Compañeras } \\
\hline Ulex baeticus subsp. baeticus & 1 & 2 & $\mathrm{x}$ \\
\hline Teucrium fruticans & 1 & 1 & $\mathrm{x}$ \\
\hline Phlomis purpurea & + & + & $\mathrm{x}$ \\
\hline Erica arborea & 1 & 3 & . \\
\hline Castanea sativa & 1 & + & . \\
\hline Cistus albidus & ; & + & $\mathrm{x}$ \\
\hline Daphne gnidium & 1 & . & $x$ \\
\hline Paeonia broteroi & . & + & $\mathrm{x}$ \\
\hline Phillyrea angustifolia & 1 & + & . \\
\hline Quercus rotundifolia & + & ; & $\mathrm{x}$ \\
\hline Quercus suber & + & 1 & . \\
\hline Rubia peregrina & + & + & . \\
\hline
\end{tabular}

Otras compañeras: Abies pinsapo x en A, Allium roseum + en 1, Asphodelus albus x en A, Brachypodium retusum + en 1, Juniperus oxycedrus x en A, Lonicera implexa + en 1, Lonicera periclymenum subsp. hispanica + en 2, Quercus faginea + en 1, Rosa micrantha + en 1, Sanguisorba hybrida 1 en 1, Thymus mastichina $\mathrm{x}$ en A y Urginea maritima + en 1.

Localidades. 1 y 2: Málaga. Parque Natural S ${ }^{a}$ de las Nieves. Parauta. Las Turquillas. UTM: UF 1761. A: Cano et al., 2003. 
que Cano et al. (op. cit.) no hacen referencia alguna a pesar de que los inventarios fueron levantados en la misma zona. Los inventarios de Cano et al. corresponden en todo caso a Cisto-Lavanduletea, puesto que sólo Lavandula lanata y Ulex baeticus indicarían la pertenencia a Ulici-Lavanduletum (Lavandulo-Echinospartion boissieri, Rosmarinetea). Ulex baeticus subsp. baeticus, Phlomis purpurea, Thymus mastichina y Cistus albidus aparecen indistintamente en jarales y matorrales y por el contrario Lavandula stoechas, Cistus ladanifer y Erica australis son especies frecuentes de Cisto-Lavanduletea. La presencia esporádica de algunas especies de Rosmarinetea (Dorycnium pentaphyllum, Staehelina dubia y Teucrium similatum) se justifica por la muy escasa potencia de estas zonas descalcificadas, rodeadas de grandes extensiones básicas (Pérez Latorre et al., op. cit.).

Por estas razones, pensamos que tanto lo descrito por Cano et al. (2003) como por Pérez Latorre et al. (1998) tratan de un único aspecto fragmentario de los jarales de Cistus laurifolius que constituyen en la Serranía de Ronda una comunidad basal en el sentido de Kopecky et al. (1995). Este tipo de hecho fitocenológico no está recogido hasta el momento por el Código Internacional de Nomenclatura Fitosociológica.

CISTO-LAVANDULETEA Br.-B1. (1940) 1952

+ Lavanduletalia stoechidis Br.-B1. 1940 em. Rivas-Martínez 1968

* Cistion laurifolii Rivas Goday in Rivas Goday et al. 1956 em. Rivas-Martínez 1979

Com. de Cistus laurifolius BC [Jarales con jara estepa]

(= Ulici baetici-Lavanduletum lanatae lavanduletosum stoechadis Cano Carmona, García Fuentes y Cano Ortíz 2003)

\section{BIBLIOGRAFÍA}

BLANCA, G., M. RUÍZ REJÓN y R. ZAMORA 1999- Taxonomic revision of the genus Pinguicula L. in the Iberian Peninsula. Folia Geobotanica 34:337-361.
CANO CARMONA, E., A. GARCÍA FUENTES \& A. CANO ORTIZ -2003- Nueva subasociación para los matorrales desarrollados sobre suelos neutros del sector rondense. Lagascalia 23: 123 132.

DÍAZ GONZÁLEZ, T. E., J. GUERRA y J. M. NIETO -1982- Contribución al conocimiento de la clase Adiantetea Br. - Bl. 1942 en la Península Ibérica. Anal. Jard. Bot. Madrid 38(2): 497-506.

HEYWOOD, V. H. -1953- El concepto de asociación en las comunidades rupícolas. Anal. Jard. Bot. Madrid 11(2): 463-481.

KOPECKY, K., J. DOSTALEK \& T. FRANTIK 1995- The use of the deductive method of syntaxonomic classification in the system of vegetational units of the Braun-Blanquet approach. Vegetatio 117: 95-112.

NIETO CALDERA, J. M. -1987-Estudio Fitocenológico de las Sierras Tejeda y Almijara. Tesis Doctoral. Universidad de Málaga.

PÉREZ LATORRE, A. V., P. NAVAS, D. NAVAS, Y. GIL y B. CABEZUDO -1998- Datos sobre la flora y vegetación de la Serranía de Ronda (Málaga, España). Acta Bot. Malacitana 23: 149-191.

RIVAS MARTÍNEZ, S., T. E. DÍAZ, F. FERNÁNDEZ GONZÁLEZ, J. IZCO, J. LOIDI y A. PENAS -2002-Vascular plant communities of Spain and Portugal. Itinera Geobotanica 15(1): 5-432.

VARO, J. y J. FERNÁNDEZ CASAS - 1970- Notas sobre algunas comunidades béticas con Adiantum capillus-veneris. Ars Pharm. 11:517520 .

Aceptado para su publicación en octubre de 2003

Dirección de los autores. Dpto. Biología Vegetal. Facultad de Ciencias. Universidad de Málaga. 29071, Málaga. 